\title{
Identification of two Mycobacterium avium subspecies paratuberculosis gene products differentially recognised by sera from rabbits immunised with live mycobacteria but not heat- killed mycobacteria
}

\author{
JOHN P. BANNANTINE* and JUDITH R. STABEL \\ National Animal Disease Center, ARS-USDA, Ames, IA 50010, USA
}

\begin{abstract}
The investigation of environmentally regulated proteins has led to a better understanding of host-pathogen interactions and identified novel vaccine candidate antigens for several bacterial pathogens. In an effort to identify such proteins in Mycobacterium avium subsp. paratuberculosis (M. paratuberculosis), a genomic expression library was differentially screened with sera from rabbits that had been immunised with live $M$. paratuberculosis ( $\alpha$-live) as well as sera from rabbits immunised with heat-killed $M$. paratuberculosis ( $\alpha$-killed). These experiments identified seven recombinant plaques that were uniquely recognised by the $\alpha$-live sera. Sequence data showed that five of these clones overlapped with each other and contained a common open-reading frame encoding a 25-kDa protein, termed Csp1. The 25-kDa antigen shows weak similarity to a secreted Corynebacterium glutamicum protein. The remaining two clones overlapped with each other and contained two partial open-reading frames, both encoding proteins with strong homology to polyketide synthase from various species of mycobacteria. Antisera were produced against a peptide of the polyketide synthase gene product designated Pks7. Csp1-specific antibodies were affinity purified from the $\alpha$-live sera. These purified antibodies demonstrated that Csp1 was present within infected macrophages. Collectively, these data identify novel $M$. paratuberculosis antigens that may be important in pathogenesis.
\end{abstract}

\section{Introduction}

Mycobacterium tuberculosis causes more deaths globally than any other infectious agent [1]. In the USA, $M$. avium complex has emerged as the most prevalent opportunist infection among patients with advanced HIV-1 infection. AIDS patients with M. avium infections suffer considerable morbidity and shortened life span $[2,3]$. Johne's disease, also called paratuberculosis, is a chronic granulomatous enteritis of ruminant animals caused by $M$. avium subsp. paratuberculosis (hereafter referred to as M. paratuberculosis). While

Received 6 Nov. 2000; revised version accepted 5 May 2001.

Corresponding author: Dr J.P. Bannantine (e-mail: jbannant@ nadc.ars.usda.gov).
Johne's disease can end in death of the animal, the economic impact of this disease is much more significant $[4,5]$.

A major effort in the study of paratuberculosis has been directed at detection of the pathogen in infected animals. In contrast, relatively little has been published on pathogenic mechanisms for paratuberculosis. The interaction between host and mycobacteria is poorly understood; almost nothing is known about the bacterial factors that enable survival of $M$. paratuberculosis within macrophages. Therefore, a significant advance in the study of host-mycobacterial interactions in Johne's disease would be the identification of $M$. paratuberculosis antigens expressed within macrophages. A few mycobacterial genes have been shown to be expressed within the macrophage for other species [6,7]. However, M. paratuberculosis sequences have not been defined in this context. This study used a 
rabbit host to identify $M$. paratuberculosis gene products that are present after entry into the animal and in cultured macrophages.

\section{Materials and methods}

\section{Construction of a genomic expression library of M. paratuberculosis}

DNA from the type strain of $M$. paratuberculosis (ATCC19698) was used to construct an expression library in a $\lambda$ phage vector (Stratagene, La Jolla, CA, USA). The size of the DNA inserts in the library ranged from 3 to $5.5 \mathrm{~kb}$, and recombinants represented $80 \%$ of the total plaques obtained. Escherichia coli XL1-Blue MRF' (Stratagene) was used as the host strain for infection with $\lambda$ ZAP express phage vector (Stratagene). Infection of $E$. coli XLOLR (Stratagene) with positive $\lambda$ clones and with ExAssist helper phage (Stratagene) resulted in the in-vivo excision of pBKCMV phagemid vector harbouring $M$. paratuberculosis inserts of interest.

\section{Rabbit sera}

Two New Zealand White rabbits were immunised with a keyhole limpet haemocyanin-conjugated peptide from the C-terminal region of the polyketide synthase (Pks7) antigen (DGPDSAPLDEDLEAATE). This peptide was chosen from algorithms that predict antigenic regions of proteins based on charge, structure and availability. Pre-immunisation sera were collected 4 days before initial immunisations. Initially, $200 \mu \mathrm{g}$ of purified KLH conjugated peptide were injected intradermally (i.d.) into each of two rabbits; 28 days later a boost of $100 \mu \mathrm{g}$ of peptide was administered subcutaneously (s.c.). A second boost was given on day 42 comprising $50 \mu \mathrm{g}$ of peptide i.d. and $50 \mu \mathrm{g}$ s.c. A final boost with $50 \mu \mathrm{g}$ of peptide delivered i.d. and s.c. was given on day 70. Test bleeds were collected by heart puncture after each booster injection. All immunisations were performed with TiterMax (CytRx, Noroross, GA, USA) as the adjuvant. Antibody responses to these immunisations were monitored by ELISA as follows. The conjugated peptide was diluted to $0.1 \mu \mathrm{g} / \mathrm{ml}$ in phosphate-buffered saline (PBS) and added to microtitration plates. The plates were incubated overnight in a humidified atmosphere at $4^{\circ} \mathrm{C}$, washed three times with PBS plus Tween $200.05 \%$ (PBST) and incubated for $30 \mathrm{~min}$ at $37^{\circ} \mathrm{C}$ with $\mathrm{PBS}$ containing gelatin $1 \%$ to block non-specific binding sites. The plates were washed three times with PBST. Test sera were diluted 1 in 200 in PBS, added to the wells and then incubated for $1 \mathrm{~h}$ at $37^{\circ} \mathrm{C}$. The plates were washed three times with PBST and alkaline phosphatase-conjugated goat anti-rabbit IgG (Pierce Chemical Company, Rockford, IL, USA) was then added for $1 \mathrm{~h}$ at $37^{\circ} \mathrm{C}$. The plates were washed three times with PBST followed by addition of $p$-nitrophenyl phosphate substrate. Negative and positive control sera were included for each assay.
The absorbances of test samples were read at $405 \mathrm{~nm}$ with a Dynatech MR7000 plate reader. Rabbits were immunised with live and heat-killed preparations of $M$. paratuberculosis as previously described [8]. The bacteria in these preparations were washed three times with PBS and resuspended to a concentration of $1 \times 10^{9}$ cells $/ \mathrm{ml}$. One preparation was killed by heating at $85^{\circ} \mathrm{C}$ for $20 \mathrm{~min}$ and the other preparation was untreated and consisted of live M. paratuberculosis.

\section{Screening of the $\lambda$ ZAP::M. paratuberculosis library}

The genomic expression library was plated on lawns of E. coli XL1-Blue MRF' and the resulting plaques were transferred to $0.01 \mathrm{M}$ IPTG-coated nitrocellulose filters (Schleicher and Schuell, Keene, NH, USA) in duplicate. One of the nitrocellulose plaque lifts from each plate was probed with serum from rabbits immunised with a live preparation of $M$. paratuberculosis ( $\alpha$-live) and the other lift was probed with serum from rabbits immunised with a heat-killed preparation of $M$. paratuberculosis ( $\alpha$-killed). Both the $\alpha$-live and $\alpha$ killed sera were pre-adsorbed with an equal volume of E. coli extract (Promega, Madison, WI, USA) $20 \mathrm{mg} / \mathrm{ml}$ for $1.5 \mathrm{~h}$ at room temperature with occasional mixing. Each pre-adsorbed serum was diluted 1 in 200 in a blocking buffer consisting of PBS with bovine serum albumin (BSA) $2 \%$ before use in immunoscreening plaques. The nitrocellulose filters were exposed to the primary antibody and processed as an immunoblot (see below). Plaques that were detected by $\alpha$-live serum, but not $\alpha$-killed serum, were selected for further study.

\section{Sequencing and DNA analysis}

Automated DNA sequencing was performed with the Taq DyeDeoxy ${ }^{\circledR}$ Terminator Cycle Sequencing Kit (Perkin Elmer/Applied Biosystems Division, San Francisco, CA, USA) on an ABI377 sequencer (Iowa State University Sequencing facility, Ames, IA, USA). Sequence assembly was performed with AssemblyLIGN ${ }^{\circledR}$ software and sequence analysis was performed with MacVector ${ }^{\circledR}$ software (Oxford Molecular, Oxford). Deduced amino acid sequences were compared with the database with the BLAST program available from the National Center for Biotechnology Information on the world-wide web. The nucleotide sequences of $M$. paratuberculosis described in this study were deposited in the GenBank Sequence Data Library, under accession numbers AF232750, AF232751 and AF232752.

\section{Protein expression}

A maltose-binding protein (MBP) fusion of Csp1 was constructed by using the pMAL-c2 vector (New England Biolabs, Beverly, MA, USA). The reading 
frame was amplified with $P$ wo polymerase (Boehringer Mannheim, Indianapolis, IN, USA) and genomic clone $270-1$ as the template. The upstream and downstream oligonucleotides for this amplification were as follows: 5'-GCGCCAGCTGATCGGTGGGGCGTGCAG-3' and 5'-GCGCAAGCTTACTGGTTCCACTTGTCGC GGATCTT-3'. This primer combination produces only the 124 internal amino acid codons from the $M$. paratuberculosis Csp1 sequence. The vector was digested sequentially with Xmn I and Bam HI, and the amplification product was digested with BamHI. Ligation of these products resulted in an in-frame fusion between the vector encoded malE gene and a portion of the cspl gene. After ligation, the products were transformed into $E$. coli DH5 $\alpha$. The insert from the construct was confirmed by DNA sequencing. The resulting fusion protein was overexpressed and purified by maltose affinity chromatography with an amylose resin (New England Biolabs). The purified MBP/Csp1 fusion protein was used as antigen to couple to an affinity column for purification of Csp1-specific antibodies.

\section{Affinity purification of antibodies}

Purified MBP/Csp1 protein was coupled by reductive amination to an agarose 4\% support column (AminoLink columns, Pierce Chemical Company, Rockford, IL, USA). The $\alpha$-live sera $(1-2 \mathrm{ml})$ were passed over the column followed by three washes and elution according to the manufacturer's instructions. Eluted fractions were evaluated by spectrophotometry. Fractions with the highest absorbance at $280 \mathrm{~nm}$ were neutralised in $1 \mathrm{M}$ Tris- $\mathrm{HCl}$ buffer $(\mathrm{pH} 9.5)$ and stored at $4^{\circ} \mathrm{C}$.

\section{Preparation of M. paratuberculosis and macrophage cell lysates for electrophoresis}

Mid-log phase Middlebrook 7H9-cultured M. paratuberculosis cells were washed twice in cold PBS and resuspended in a minimal amount of PBS. This suspension was sonicated on ice at $90 \mathrm{~W}$ for three 10-min intervals. The sonicated lysate was assayed for protein content (BioRad protein assay), diluted to $1 \mathrm{mg} / \mathrm{ml}$ in PBS, and stored frozen at $-20^{\circ} \mathrm{C}$. Frozen aliquots were thawed, mixed with $1 \times$ SDS loading buffer (sodium dodecyl sulphate 1\%,50 mM Tris, $\mathrm{pH}$ 6.8, 2-mercaptoethanol 1\%, glycerol 10\%) and boiled for $5 \mathrm{~min}$ before loading on to SDS polyacrylamide gels. Bovine macrophages (BoMacs [9]) were grown in RPMI 1640 medium supplemented with fetal calf serum 10\%. Murine J774.16 macrophages were cultured in Dulbecco's minimal essential medium supplemented with fetal calf serum 7.5\%. All macrophage cultures were grown at $37^{\circ} \mathrm{C}$ in $\mathrm{CO}_{2} 5 \%$. Opsonised $M$. paratuberculosis was used to infect macrophages at between 10:1 and 50:1 mycobacteria:macrophage. After a 4-h infection period on a rocker platform, remaining extracellular bacteria were removed and monolayers were washed twice with PBS before adding culture media. Infected and mock-infected macrophage monolayers in six-well trays were harvested between 2 and 4 days after infection by washing briefly with Hanks's Balanced Salts Solution (Life Technologies, Gaithersburg, MD, USA) followed by lysis with $1 \times$ SDS loading buffer $500 \mu \mathrm{l} /$ well. Lysates were mixed and distributed into $1.5-\mathrm{ml}$ microcentrifuge tubes before boiling for $5 \mathrm{~min}$. Lysates were stored at $-20^{\circ} \mathrm{C}$.

\section{Electrophoresis and immunoblot analysis}

$E$. coli lysates were prepared as described previously [10]. Polyacrylamide gel electrophoresis was performed with polyacrylamide $12 \% \mathrm{w} / \mathrm{v}$ gels. Electrophoretic transfer of proteins on to pure nitrocellulose (Schleicher and Schuell, Keene, NH, USA) was accomplished with the BioRad Trans Blot Cell (BioRad Laboratories, Richmond, CA, USA) with sodium phosphate buffer (25 mM, pH 7.8) at $0.8 \mathrm{~A}$ for $90 \mathrm{~min}$. After transfer, filters were blocked with phosphate-buffered saline (PBS; $150 \mathrm{~mm} \mathrm{NaCl}, 10 \mathrm{mM} \mathrm{NaPO}_{4}, \mathrm{pH} 7.4$ ) plus BSA $2 \%$ and Tween $200.1 \%$ (PBS-BSA). Antisera were diluted 1 in 1000 in PBS-BSA and incubated on the blot at room temperature for $2 \mathrm{~h}$. After three washes in PBS plus Tween-20 0.1\% blots were incubated for $1.5 \mathrm{~h}$ in protein A-peroxidase (Pierce) diluted 1 in 15000 in PBS-BSA. The blots were again washed three times as described above and developed for chemiluminesence with Supersignal detection reagents (Pierce).

\section{Immunofluorescence microscopy of infected cells}

Indirect fluorescent antibody (FA) microscopy was performed on infected and mock-infected macrophages grown as monolayers on sterile glass chamber slides (Nalge Nunc International). Macrophages to be immunostained were washed with PBS and fixed with methanol $100 \%$ between 1 and 3 days after infection. Fixed cells were washed with PBS after the 5-min methanol treatment and stored at $4^{\circ} \mathrm{C}$ in PBS.

Slides containing cells were first immersed in PBSBSA (without Tween-20) for 5-10 min at room temperature. Affinity purified antibodies were then added at a 1 in 400 dilution in PBS-BSA (without Tween 20). Macrophages were incubated on a rocker platform at room temperature for $1 \mathrm{~h}$ and then washed three times with PBS. Fluorescein-labelled goat anti-rabbit immunoglobulin $\mathrm{G}$ (Pierce) was added at a dilution of 1 in 500 in PBS-BSA (without Tween 20) and then incubated in the dark for another hour. Immunostained cells were washed again three times with PBS and mounted for microscopy using Vectashield (Vector Laboratories) mounting medium. Labelled cells were visualised with a Nikon E800 series microscope with a FITC cube and DIC optics. 


\section{Results}

Identification of antigens present uniquely within infected macrophages by immunoblot analysis with $\alpha$-live sera

Two preparations of antisera were produced in New Zealand White rabbits. One group of three rabbits was immunised with live M. paratuberculosis and a second group of three was immunised with heat-killed $M$. paratuberculosis. When immunoblots of $M$. paratuberculosis-infected BoMac macrophage lysates were probed with sera from rabbits immunised with live M. paratuberculosis ( $\alpha$-live), a set of proteins was identified that were not present in uninfected macrophages or Middlebrook 7H9-cultured M. paratuberculosis (Fig. 1). Two of the most predominant protein bands, migrating at 55 and $39 \mathrm{kDa}$ and present only within infected macrophages, are indicated with arrows in Fig. 1. Conversely, an identical immunoblot probed with sera from rabbits immunised with heat-killed $M$. paratuberculosis ( $\alpha$-killed) did not detect these same antigens. Based upon this initial observation, a search for $M$. paratuberculosis antigens expressed within the macrophage was undertaken.

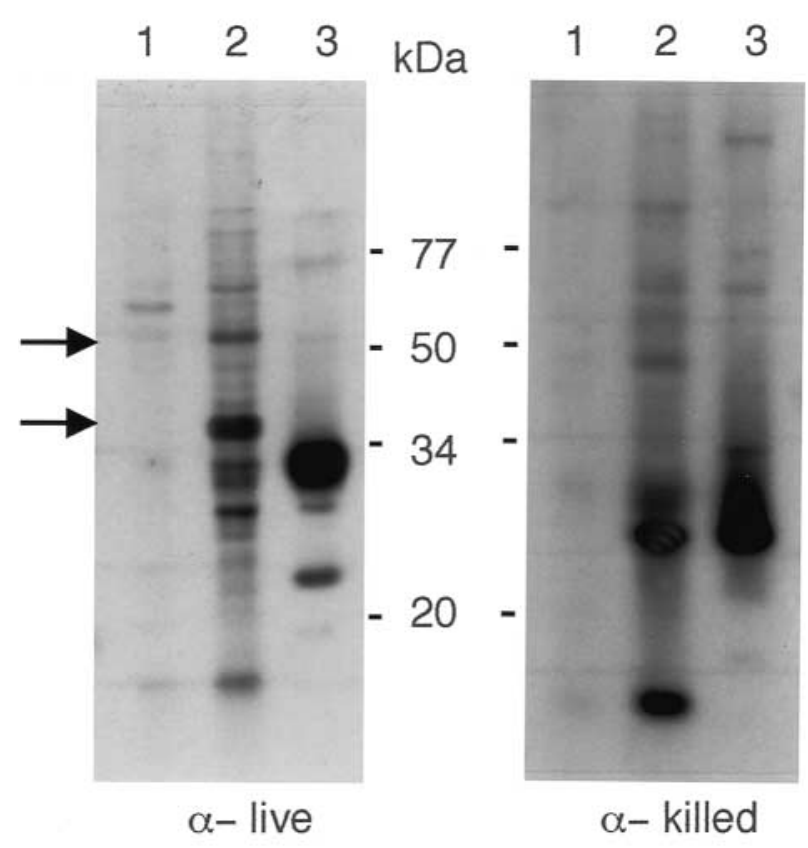

Fig. 1. At least two proteins were detected uniquely within $M$. paratuberculosis-infected macrophage lysates when probed with $\alpha$-live sera. Two identical immunoblots of infected BoMac macrophages are shown. Lane 1, mock-infected macrophages; 2, M. paratuberculosisinfected macrophages (3 days after infection); 3, $M$. paratuberculosis cultured in Middlebrook $7 \mathrm{H} 9$ medium. One immunoblot was probed with serum from rabbits immunised with live $M$. paratuberculosis ( $\alpha$-live) and the other with serum from rabbits immunised with heatkilled M. paratuberculosis ( $\alpha$-killed) sera. Size standards in $\mathrm{kDa}$ are shown between the blots and arrows in the left margin indicate proteins present only within infected macrophages (lane 2). These same proteins were not detected in the immunoblot probed with $\alpha$-killed sera.

\section{Identification of immunoreactive library clones}

The strategy to identify $M$. paratuberculosis antigens expressed within the macrophage was to screen an expression library with $\alpha$-live and $\alpha$-killed sera. Duplicate nitrocellulose lifts were produced for $c$. 150000 M. paratuberculosis library plaques. One lift from each plate was probed with $\alpha$-live sera and the other lift was exposed to $\alpha$-killed sera. Several plaques were detected by both sera and 36 plaques were detected by the $\alpha$-killed sera alone. Only seven plaques showed differential immunoreactivity with the $\alpha$-live but not $\alpha$-killed sera. DNA inserts from these seven recombinant phage clones were subcloned into $\mathrm{pBK}$ CMV by in-vivo excision. SDS-PAGE and immunoblot analysis of E. coli harbouring the mycobacterial inserts from four of the seven library clones showed that each produced a protein that was recognised by the $\alpha$-live sera, but not by $\alpha$-killed sera (Fig. 2). Clones 270-1, 270-2 and 270-3 all produced a similar sized $25-\mathrm{kDa}$ protein, whereas clone $270-4$ produced a $70-\mathrm{kDa}$ protein. Clone 275-1, which was detected only by the $\alpha$-killed sera, was processed in parallel and served as a control. Both clones 270-1 and 275-1 were expressed by $E$. coli regardless of the presence or absence of IPTG.

\section{Sequence analysis}

All clones that showed reactivity solely with the $\alpha$-live sera were sequenced. Five of the clones, designated 270-1, 270-2, 270-3, 270-5 and 270-6, overlapped with each other and the remaining two (270-4 and 270-7) aligned with each other as shown in Fig. 3a. It should be noted that all clones were overlapping and not identical siblings, therefore a $5.5-\mathrm{kb}$ contiguous sequence was identified surrounding cspl and a 4-kb region was assembled from the 270-4 and 270-7 clones. The open-reading frames (ORFs) whose gene products were detected by the $\alpha$-live sera are highlighted. The function of its gene product (designated Csp1) is unknown, although it does contain similarity to a secreted protein from Corynebacterium glutamicum also termed Csp1 [11]. The E value for this comparison by BLAST analysis is 1e-10.

ORF maps of the assembled clones show that cspl is immediately downstream from $\mathrm{purH}$, which encodes a formyltransferase (Fig. 3b). The pks7 sequence is situated upstream of another polyketide synthase, designated pks8. A homology search of this sequence with the genetically similar $M$. avium genome revealed another polyketide synthase-encoding sequence upstream of $p k s 7$ as well. The 275-1 ORF map was the control clone used in the experiment shown in Fig. 2. The major membrane protein-1 gene product was detected by the $\alpha$-killed sera and not by $\alpha$-live sera.

In total, over $12 \mathrm{~kb}$ of $M$. paratuberculosis sequence was produced in this study and all of it aligned with $M$. 


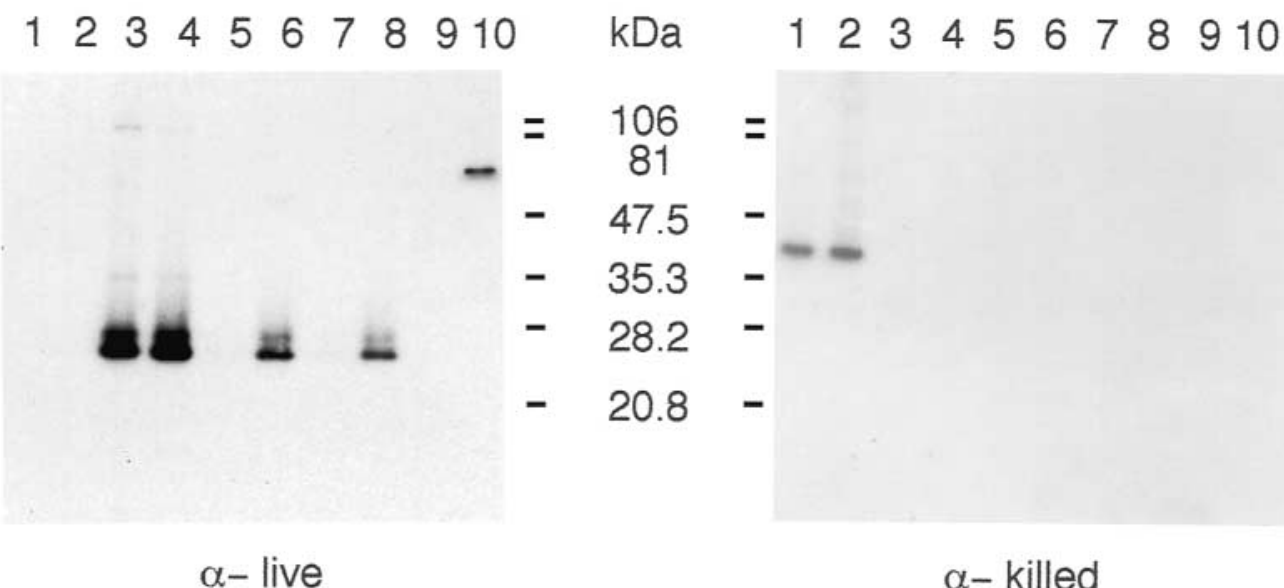

Fig. 2. Immunoblot analysis of $M$. paratuberculosis genomic library clones showing differential reactivity with the $\alpha$ live and $\alpha$-killed sera. Two identical immunoblots are shown loaded with E. coli lysates harbouring positive clones. One immunoblot was probed with $\alpha$-live serum and the other with $\alpha$-killed serum. Clone 275-1 was processed in parallel as a control that reacted uniquely with the $\alpha$-killed serum. Lane 1, clone 275-1 without IPTG; 2, clone 275-1 with IPTG; 3, clone 270-1 without IPTG; 4, clone 270-1 with IPTG; 5, clone 270-2 without IPTG; 6, clone 270-2 with IPTG; 7, clone 270-3 without IPTG; 8, clone 270-3 with IPTG; 9, clone 270-4 without IPTG; 10, clone 270-4 with IPTG. Size standards in $\mathrm{kDa}$ are shown between the blots.

avium sequences. Of the 4844 nucleotides in clone 270-1, only 29 did not match. Furthermore, there was one nine-nucleotide stretch of sequence present in $M$. avium that was not in $M$. paratuberculosis. These collective differences resulted in a 99\% nucleotide identity with $M$. avium over the $4.8-\mathrm{kb}$ sequence. The same percentages were observed for clones 275-1 and 270-4.

\section{Expression of ORFs detected by $\alpha$-live sera}

Because multiple ORFs were present in the library clones, it could not be unequivocally determined that Csp 1 and Pks 7 were detected by $\alpha$-live sera solely by sequence analysis. Therefore, cspl was expressed in $E$. coli as a fusion protein and analysed by immunoblot (Fig. 4a). The SDS-PAGE gel shows the positions and relative amounts of purified protein present in each immunoblot. The blot probed with $\alpha$-live sera detected only the MBP/Csp1 fusion protein and not MBP alone, whereas the $\alpha$-killed sera recognised neither protein (Fig. 4a). To identify which $p k s$ sequence encoded the protein detected by $\alpha$-live sera, an immunoblot was performed with the 270-4 and 270-7 library clone lysates (Fig. 4b). As shown in Fig. 3a, more of the pks 7 ORF was present in clone 270-4 than in clone 270-7. Therefore, immunoblot analysis with antisera that recognise the pks 7 gene product should detect a larger protein band in clone 270-4 than in clone 270-7. This is exactly what was observed experimentally when the immunoblot was probed with $\alpha$-live sera (Fig. 4b). No reactivity was observed when an identical blot was probed with $\alpha$-killed sera (Fig. 4b). Based on these experiments, it is clear that Csp1 and Pks7 were detected uniquely by $\alpha$-live sera.
Antibody was produced against a KLH conjugated peptide designed from the Pks7 sequence. The resulting antibody showed weak reactivity by immunoblot of $E$. coli lysates harbouring clone 270-4 (Fig. 5). Csp1specific antibodies were purified from $\alpha$-live sera by affinity chromatography. Eluted antibody showed strong reactivity with the 270-1 clone (data not shown).

\section{Determination of Csp1 within infected macrophages by immunoblot and immunofluorescent microscopy analysis with specific antibody}

M. paratuberculosis bacilli were grown under two different environmental conditions: in Middlebrook $7 \mathrm{H} 9$ broth at $37^{\circ} \mathrm{C}$ and in macrophages derived from bovine peripheral blood monocytes or established cell lines such as J774 macrophages and BoMac cells [9]. Protein lysates were prepared from each culture condition and analysed by SDS-PAGE and immunoblot with affinity-purified Csp1-specific antibody. Despite affinity purification, Csp1 antibodies detected multiple J774 macrophage proteins, as shown in lanes 1 and 2 of Fig. 6. However, Csp1 was detected in $M$. paratuberculosis lysates as indicated by the $25-\mathrm{kDa}$ band present in lane 3 of Fig. 6 . This same $25-\mathrm{kDa}$ band was also present in infected macrophages (lane 2 of Fig. 6). With the same methodology, Csp1 was also detected in infected BoMac macrophages (data not shown).

Methanol-fixed M. paratuberculosis-infected J774 and BoMac macrophages were immunostained with affinity-purified antibodies and visualised by immunofluorescence microscopy. Antibody produced against a 

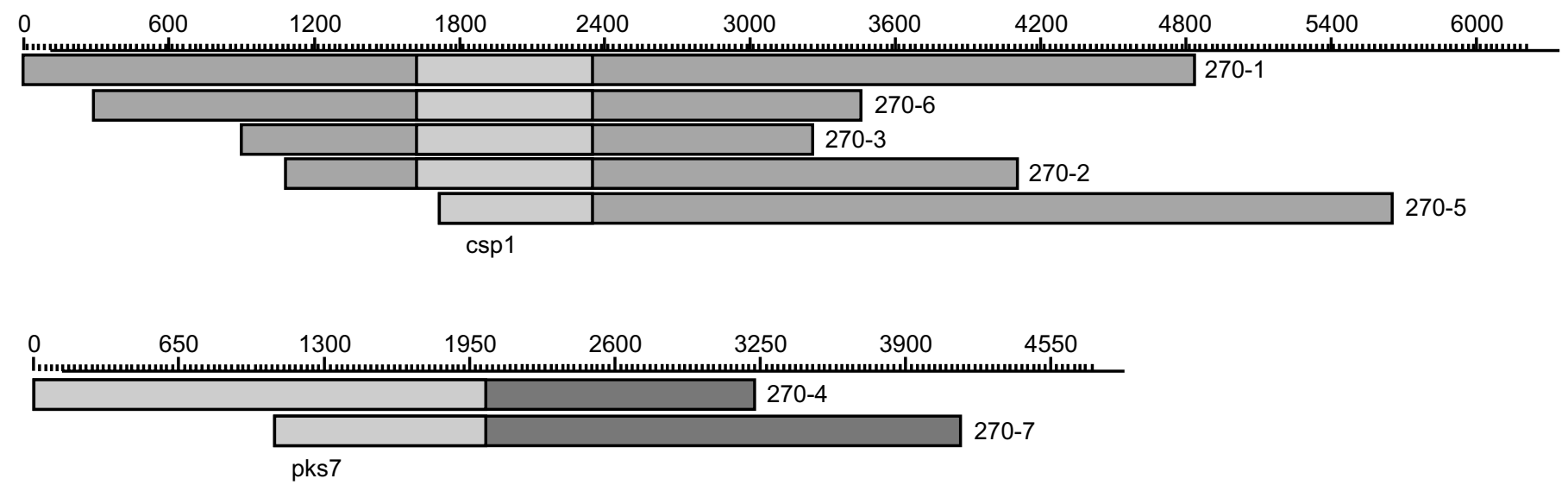

b

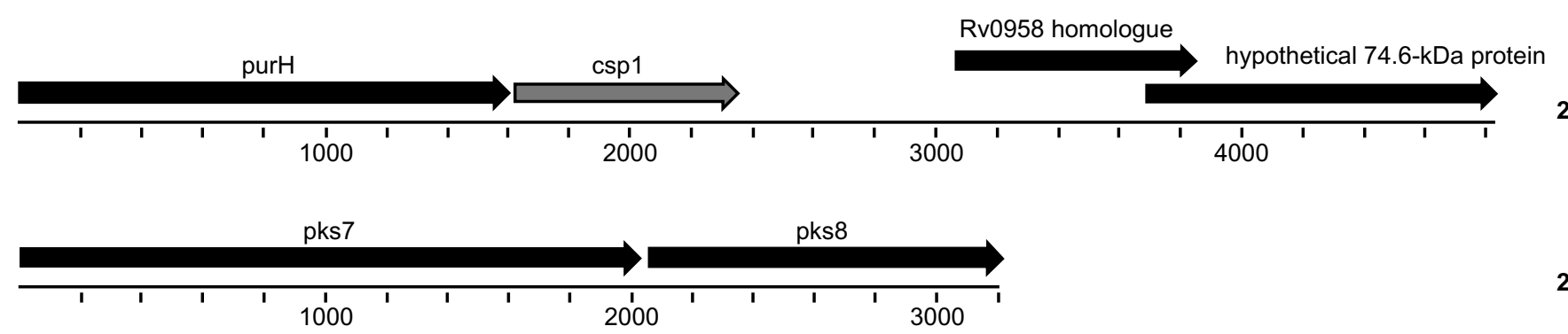

270-1

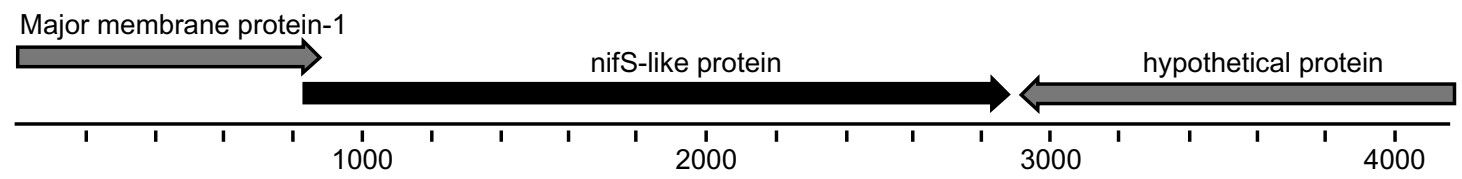

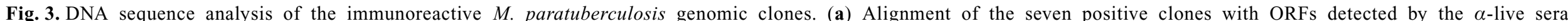

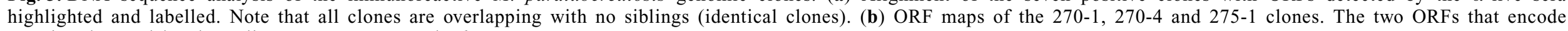
proteins detected by the $\alpha$-live sera are $\operatorname{csp} 1$ and $p k s 7$. 
a

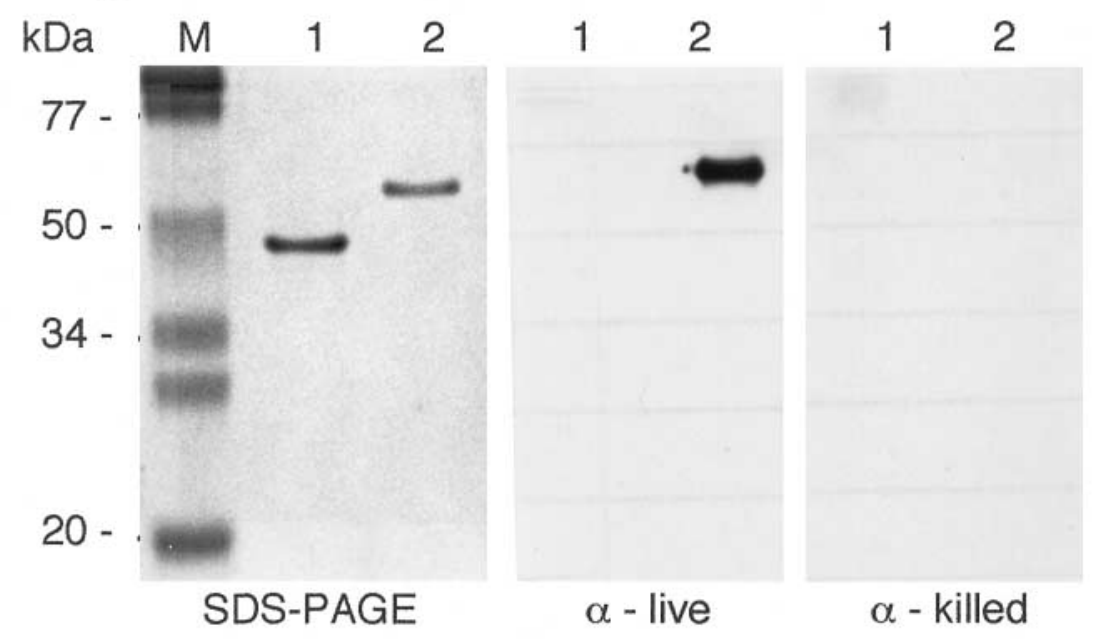

b
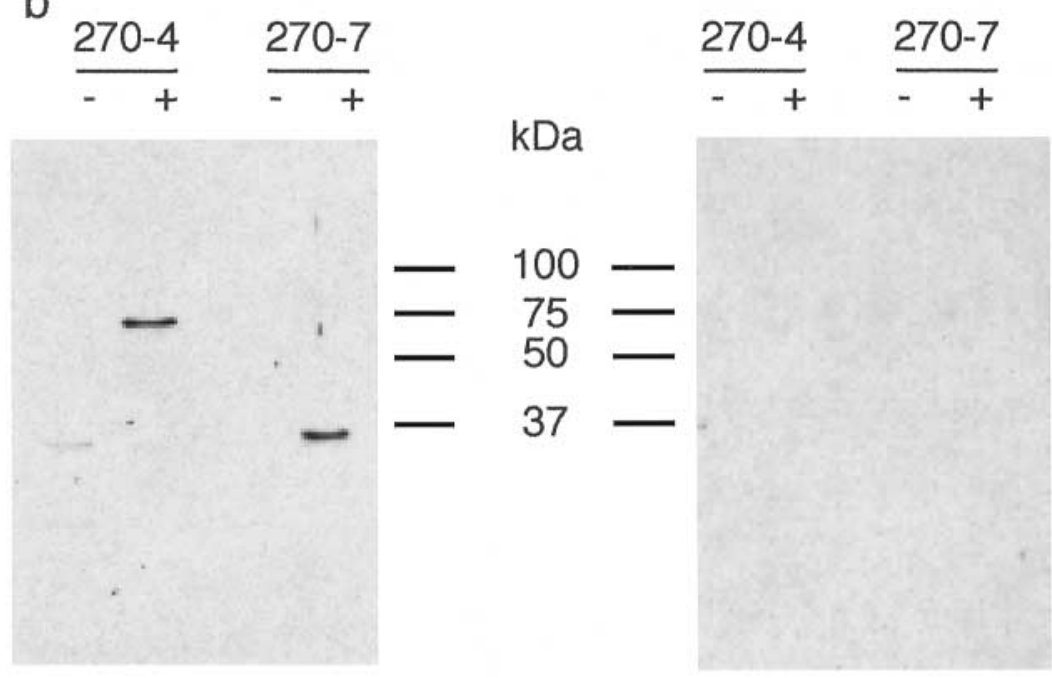

$\alpha$ - live

$\alpha-$ killed

Fig. 4. MBP/Csp1 fusion protein and Pks7 detected only by the $\alpha$-live sera. An SDS-PAGE gel and two corresponding immunoblots are shown. (a) The SDS-PAGE gel identifies the positions of MBP (lane 1) and MBP/Csp1 (lane 2). The two immunoblots show that MBP/Csp1, not MBP alone, is detected uniquely by $\alpha$-live sera. Lane M, molecular size markers, some of which are listed in the left margin in kDa. (b) Identical immunoblots of the 270-4 and 270-7 library clones probed with $\alpha$-live and $\alpha$-killed sera. The lane assignments are indicated above each blot. The + or designation indicates the presence or absence of IPTG in the culture. Size standards in $\mathrm{kDa}$ are shown in the left margin.

peptide of Pks7 did not yield a high enough titre to conclusively demonstrate the presence of Pks7 within infected macrophages. The Csp1 antibodies labelled $M$. paratuberculosis within both macrophage cell lines (Fig. 7), but showed no reactivity with uninfected macrophages (black arrow in $7 \mathrm{a}$ and c). As with all infected macrophages used in this study, extracellular mycobacteria were washed off as described in Methods. Therefore, only intracellular bacteria were stained in this assay. Csp1 was never detected in uninfected macrophages either by immunoblot or immunofluorescence microscopy. Collectively, these data provided convincing evidence that Csp1 was present within infected, cultured macrophages.

\section{Distribution of Csp1}

Because of the similarity between Csp1 and a major secreted protein in C. glutamicum, it was of interest to determine if Csp1 is secreted in M. paratuberculosis. Sequence analysis identified no known signal peptidase cleavage site. The method of Mutharia et al. [12] was used to capture M. paratuberculosis proteins secreted in the Middlebrook $7 \mathrm{H} 9$ culture medium as well as 


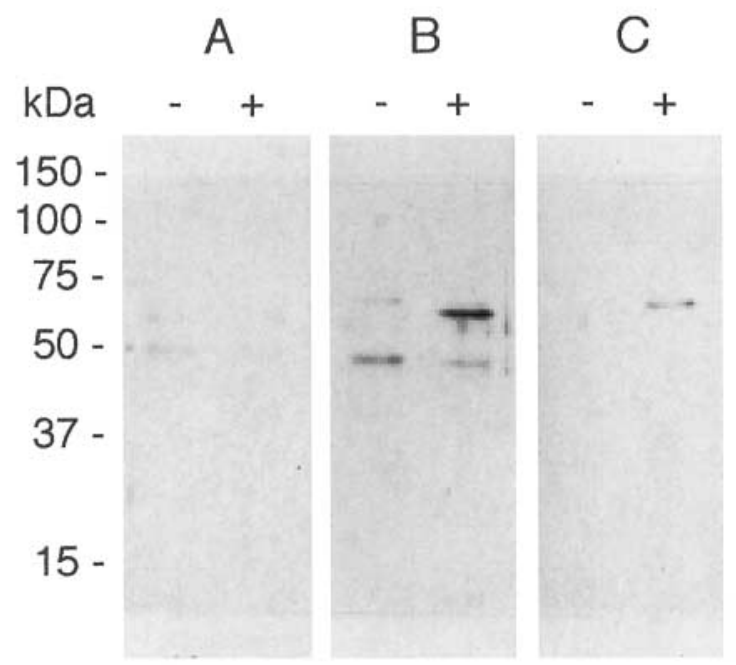

Fig. 5. Pks7 peptide antibody detection of the recombinant antigen expressed in clone 270-4. Three identical immunoblots of the 270-4 E. coli clone are shown. One was probed with pre-immunisation sera $(\mathbf{A})$, another was probed with post-immunisation sera (B) and a third was probed with $\alpha$-live sera $(\mathbf{C})$. The $+/-$ designations above the blots indicate the presence or absence of IPTG in the culture. Size standards in $\mathrm{kDa}$ are shown in the left margin. a

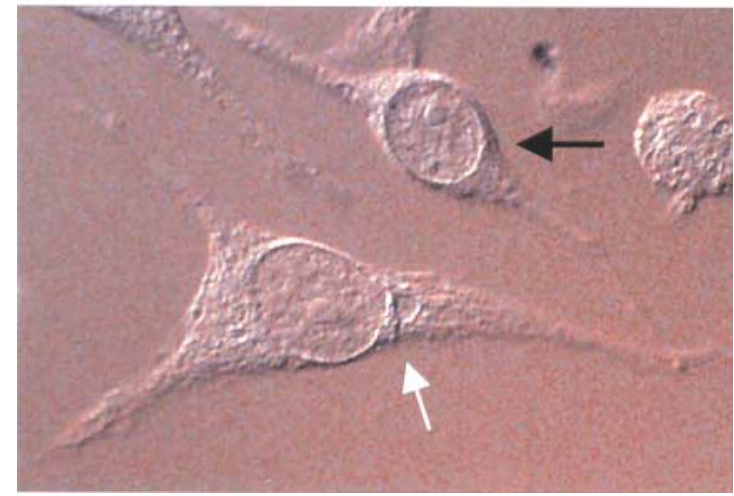

b

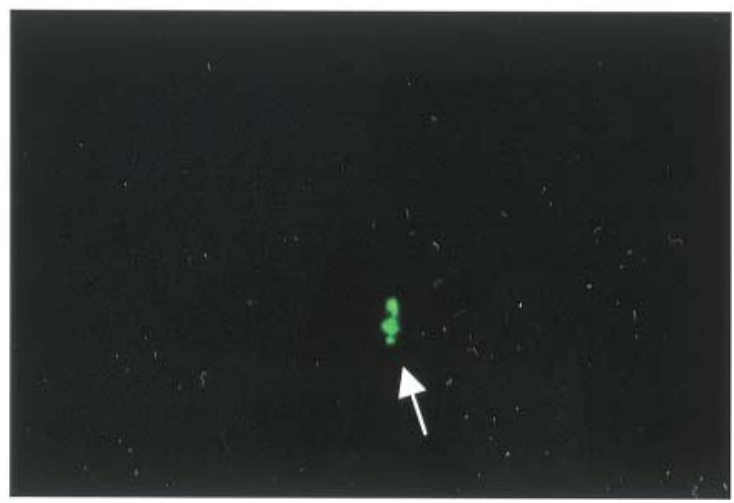

C

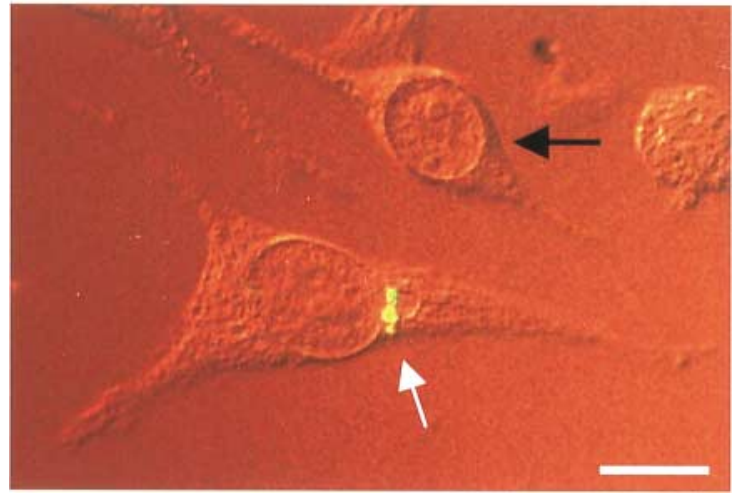

Fig. 7. Immunofluorescence microscopy showing Csp1 within $M$. paratuberculosis-infected J774 macrophages. M. paratuberculosis-infected macrophages were fixed with methanol at $54 \mathrm{~h}$ after infection. Fixed cells were immunostained with purified Csp1 antibodies as described in Materials and methods. Differential interference contrast (a), immunofluorescent (b) and merged (c) images of an $M$. paratuberculosis-infected macrophage show labelling of the bacteria within the macrophage. The black arrow identifies an uninfected macrophage that shows no reactivity and the white arrow shows the location of the mycobacteria within each image. Bar in panel c, $10 \mu \mathrm{m}$. 
produce a lysate of soluble cell extracts. Immunoblots of these separated fractions probed with Csp1 antibody showed that Csp1 was present only in the soluble cell extract and was not secreted into the medium (data not shown).

\section{Discussion}

Immunoblot analysis has shown, for the first time, proteins present uniquely during $M$. paratuberculosis infection of cultured macrophages when probed with sera from rabbits immunised with live $M$. paratuberculosis. These data prompted a search for M. paratuberculosis proteins present within infected macrophages. Two pathogen-derived proteins, Csp1 and Pks7, were identified from seven library clones that reacted uniquely with $\alpha$-live sera. Csp1 was also shown to be present within infected macrophages, but not exclusively, as it was also present in protein lysates from mycobacteria cultured in standard laboratory media. The fact that this differential screening strategy identified multiple overlapping library clones that contained Csp1- and Pks7-encoding sequences indicates a well represented library. There is little doubt that these antigens are recognised only when live mycobacteria are present within the rabbit host. Signs of Johne's disease have been produced in rabbits infected with $M$. paratuberculosis $[13,14]$ and naturally infected rabbits have been documented in Scotland [15]. Therefore, a rabbit model may be useful for studying Johne's disease. A caveat to this model system is that the rabbit hosts were not naturally infected with paratuberculosis organisms but were infected by injection at various sites. Studies are planned to determine why these antigens are differentially present in rabbits.

In recent years, considerable attention has been given to secreted proteins which are probably available for presentation to the immune system before cytoplasmic constituents, which depend on lysis of the bacterial cell for presentation. Therefore, secreted proteins are expected to be of particular importance for immune responses immediately after infection and are of great interest in the development of new vaccines against mycobacteria. Csp1 bears weak homology to a major secreted protein of C. glutamicum [11]. However, no known signal peptidase cleavage sites are present in $M$. paratuberculosis Csp1. Furthermore, it was found in this study that Csp1 was contained within the soluble cell extract, suggesting that this protein is not secreted by $M$. paratuberculosis in vitro.

A search for polyketide synthase sequences in the $M$. avium and $M$. tuberculosis genomes reveals that each species contains a large family of these sequences. $M$. tuberculosis, for example, has no less than 18 polyketide synthase-encoding sequences [16]. Therefore, a high percentage of metabolic energy and genomic sequence is devoted to the synthesis of polyketides. Polyketides are lipid-like molecules that have potent biological activities. Well-known polyketides include antibiotics (erythromycin), antifungal agents (amphotericin B), immunosuppressants (FK506) and cytostatins (bafilomycin). Mycobactins are produced in $M$. tuberculosis by means of a polyketide synthase/non-ribosomal synthase strategy [17]. Mycolactone, the first described mycobacterial toxin [18], is also a polyketide compound, which may implicate polyketide synthase as a virulence protein.

Identification of potential virulence determinants has been very difficult due to the lack of well-developed genetic systems in this slow-growing pathogen. This situation should improve when the genome sequence of M. paratuberculosis is obtained. Armed with the genomic sequence, investigators can then probe this pathogen's global gene expression under a specified set of physiological conditions, such as those found within the infected macrophage.

The nucleotide identity between the $M$. paratuberculosis sequences described here and M. avium is striking at $99 \%$ The discovery of regions unique to each genome may soon emerge once the sequencing phase is complete. Those unique regions may well hold the key to the divergent clinical diseases caused by each organism.

The expert technical assistance of Janis Hansen and Trudy Bosworth is gratefully acknowledged.

\section{References}

1. Dye C, Scheele S, Dolin P, Pathania V, Raviglione MC. Consensus statement. Global burden of tuberculosis: estimated incidence, prevalence, and mortality by country. WHO Global Surveillance and Monitoring Project. JAMA 1999; 282: $677-686$.

2. Bloom BR, Murray CJL. Tuberculosis: commentary on a reemergent killer. Science 1992; 257: 1055-1064.

3. Jacobson MA, Hopewell PC, Yajko DM et al. Natural history of disseminated Mycobacterium avium complex infection in AIDS. J Infect Dis 1991; 164: 994-998.

4. Ott SL, Wells SJ, Wagner BA. Herd-level economic losses associated with Johne's disease on US dairy operations. Prev Vet Med 1999; 40: 179-192.

5. Johnson-Ifearulundu Y, Kaneene JB, Lloyd JW. Herd-level economic analysis of the impact of paratuberculosis on dairy herds. J Am Vet Med Assoc 1999; 214: 822-825.

6. Arruda S, Bomfim G, Knights R, Huima-Byron T, Riley LW. Cloning of an $M$. tuberculosis DNA fragment associated with entry and survival inside cells. Science 1993; 261: 1454-1457.

7. Plum G, Clark-Curtiss JE. Induction of Mycobacterium avium gene expression following phagocytosis by human macrophages. Infect Immun 1994; 62: 476-483.

8. Stabel JR, Ackermann MR, Goff JP. Comparison of polyclonal antibodies to three different preparations of Mycobacterium paratuberculosis in immunohistochemical diagnosis of Johne's disease in cattle. J Vet Diagn Invest 1996; 8: 469-473.

9. Stabel JR, Stabel TJ. Immortalization and characterization of bovine peritoneal macrophages transfected with SV40 plasmid DNA. Vet Immunol Immunopathol 1995; 45: 211-220.

10. Rockey DD, Rosquist JL. Protein antigens of Chlamydia psittaci present in infected cells but not detected in the infectious elementary body. Infect Immun 1994; 62: 106-112.

11. Joliff G, Mathieu L, Hahn V et al. Cloning and nucleotide sequence of the csp1 gene encoding PS1, one of the two major 
secreted proteins of Corynebacterium glutamicum: the deduced N-terminal region of PS1 is similar to the Mycobacterium antigen 85 complex. Mol Microbiol 1992; 6: 2349-2362.

12. Mutharia LM, Moreno W, Raymond M. Analysis of culture filtrate and cell wall-associated antigens of Mycobacterium paratuberculosis with monoclonal antibodies. Infect Immun 1997; 65: 387-394.

13. Mokresh AH, Czuprynski CJ, Butler DG. A rabbit model for study of Mycobacterium paratuberculosis infection. Infect Immun 1989; 57: 3798-3807.

14. Mokresh AH, Butler DG. Granulomatous enteritis following oral inoculation of newborn rabbits with Mycobacterium paratuberculosis of bovine origin. Can J Vet Res 1990; 54: 313-319.
15. Greig A, Stevenson K, Henderson D et al. Epidemiological study of paratuberculosis in wild rabbits in Scotland. J Clin Microbiol 1999; 37: 1746-1751.

16. Cole ST, Brosch R, Parkhill J et al. Deciphering the biology of Mycobacterium tuberculosis from the complete genome sequence. Nature 1998; 393: 537-544.

17. Quadri LE, Sello J, Keating TA, Weinreb PH, Walsh CT. Identification of a Mycobacterium tuberculosis gene cluster encoding the biosynthetic enzymes for assembly of the virulence-conferring siderophore mycobactin. Chem Biol 1998; 5: 631-645.

18. George KM, Chatterjee D, Gunawardana G et al. Mycolactone: a polyketide toxin from Mycobacterium ulcerans required for virulence. Science 1999; 283: 854-857. 